\title{
Navigating and interacting indoors with a mobile learning game
}

\author{
Schwabe, Gerhard ; Göth, Christoph
}

\begin{abstract}
The paper reports on the experiences of designing the interface of a mobile learning game. Three system versions were presented to discuss design issues and the results of three trials (the largest with more than 100 users). Eight design implications resulted from those trials: 1. Base the navigation interface on maps designed for walking. 2. Precise zooming is adequate for standing use only; button based coarse zooming is adequate for walking. 3. Good navigation support does not only cover the current situation, but also captures the past and supports planning. 4. Visualise imprecision of location information appropriately. 5. Chat-based communication is inadequate for many game situations. Carefully designed oral communication would be more appropriate in many situations. 6. Embed competitive awareness into the other channels. 7. Design explicitly for standing and walking use. 8. Design systems for user pairs. The design approaches discussed in this paper can also be applied to other mobile applications such as mobile learning in museums, emergency support or tourism.
\end{abstract}

DOI: https://doi.org/10.1109/WMTE.2005.47

Posted at the Zurich Open Repository and Archive, University of Zurich ZORA URL: https://doi.org/10.5167/uzh-61367

Conference or Workshop Item

Originally published at:

Schwabe, Gerhard; Göth, Christoph (2005). Navigating and interacting indoors with a mobile learning game. In: International Workshop on Wireless and Mobile Technologies in Education, Tokushima, 28 November 2005 - 30 November 2005, IEEE.

DOI: https://doi.org/10.1109/WMTE.2005.47 


\title{
Navigating and interacting indoors with a mobile learning game
}

\author{
Gerhard Schwabe, Christoph Göth, University of Zurich, Switzerland, schwabe@ifi.unizh.ch
}

\begin{abstract}
The paper reports on the experiences of designing the interface of a mobile learning game. Three system versions were presented to discuss design issues and the results of three trials (the largest with more than 100 users). Eight design implications resulted from those trials: 1. Base the navigation interface on maps designed for walking. 2. Precise zooming is adequate for standing use only; button based coarse zooming is adequate for walking. 3. Good navigation support does not only cover the current situation, but also captures the past and supports planning. 4. Visualise imprecision of location information appropriately. 5. Chat-based communication is inadequate for many game situations. Carefully designed oral communication would be more appropriate in many situations. 6. Embed competitive awareness into the other channels. 7. Design explicitly for standing and walking use. 8. Design systems for user pairs. The design approaches discussed in this paper can also be applied to other mobile applications such as mobile learning in museums, emergency support or tourism.
\end{abstract}

\section{Introduction}

Mobile games have a high potential for improved learning. The participants experience immersion in a mixed reality environment, particularly due to the navigational features. While we have establish these motivational effects in a prior publication [1], this paper focuses on how such an experience can be designed. Rather than focussing on the general architecture of the system (already covered in $[2,3]$ ), we explore the user interface of the system. The users of the game have to navigate in physical space under time pressure. How can one achieve immersion for such an environment? The insights reported here were gained during two years of developing and testing MobileGame as part of the EU-project Mobilearn [4].

The next section will briefly introduce MobileGame, its design philosophy, its architecture and its three main interface versions. Section 3 reports on the research, design and data collection methods. The subsequent two sections analyse user trials and their interface design implications. Section 4 discusses map navigation and section 5 focuses on the usage and design of different interaction channels. The final section summarises our findings and proposes future research.

\section{Game}

\subsection{Description Scenario}

MobileGame is used to support the orientation days at a university. The traditional orientation rally is electronically supplemented with handheld devices. The orientation rally is a fun event aimed at familiarising the students with the university and its surroundings. Therefore, the rally will lead all participants through an area with several tasks to carry out at certain spots. The students play individually or in small teams (2-4 persons) against each other or against other teams ${ }^{1}$. Each individual /team receives a handheld computer.

During the orientation rally, each team receives different tasks referring to significant places, people and events (explained below). The handheld device shows the current position of the team on the digital map of the university. When the team enters a building, the outdoor map switches to an indoor map of the building the team just entered. The whole rally is structured as a co-operative and competitive game. Competition is based on hunting rules: Each team tries to catch another team and, equally, is hunted by a third team ${ }^{2}$. The handheld device shows each team where its hunter and its prey are located. Cooperation rules force team members to meet members from other teams as well as teachers and to exchange information with them - again they are supported with location based information on their displays. The tasks provide them with basic information on university life. The types of tasks are as follows:

- Significant place tasks: The students have to find important places, such as the library, the cafeteria or the laboratories. At each location, they have to perform a typical task (find a book, have lunch, etc.). The specific tasks are context-dependent (they depend not only on the location, but also on the time of the day or they build on the activity of some previous team). The handheld device supports the task execution (e.g. serving as a front-end to the library information system or providing needed information).

- Significant people tasks: The students have to find important people of the university and have to interview

\footnotetext{
${ }^{1}$ In order to simplify the text, this scenario assumes that a there is a team of players.

2 The didactic reason for hunting is to keep the groups moving. Of course, there need to be hunting free areas and times, e.g. during lectures/seminars.
} 
them on their activities (the president, the study coordinator, the caretaker, etc). These people either participate in the game or are played by elder students). If important people are typically mobile a mobile device can be used to locate them.

- Significant event tasks: The significant events can be scheduled or come as surprise. Scheduled events include introductory lectures and courses. Here, tasks relate to the organisation of studies (e.g. seting up a course schedule, or how to find important information) and some initial content. Unscheduled events include both "spontaneous" welcome parties by student groups, and also the signup of each team member to important university services (e.g. computer account, library card).

Each task requires the team to answer one or two simple questions displayed on the handheld device. For example, one task might be to find the cafeteria. Once there, they must answer the question "What is the price of an apple pie?" correctly before they get the next task.

The described scenario is typical for mobile learning: While the potential for using mobile learning in classical teaching is limited, it opens the arena for non-standard learning such as informal learning, ad-hoc learning and situated learning. In this scenario the participants learn basic student skills such as using the library and finding their ways to lecture halls.

\subsection{Design approach and system architecture}

When developing the MobileGame we strove to understand mobile learning and how to best design a system to support it. This leads to a design approach different to most prior research on indoor navigation. Rather than concentrating on optimising specific features or testing a certain technology, we aimed at optimising fun and learning experience. This approach requires a satisfactory and balanced design solution to all-important aspects of the game as a single weakness could ruin the experience. In order to achieve this objective in reasonable time, we used off the shelf technology wherever possible. Off the shelve technology may not include all innovative features of pure research prototypes and it may restrict the design, but it is reliable, scalable and of tested usability, allowing to build a rich trial environment. The research value of this is approach is twofold:

1. It provides a comprehensive picture of design choices based on a comparatively large set of user data. The comprehensive picture includes issues of balancing the parts of the system.

2. It shows what can be done using state-of-the-art technology. Thus the results can be used by others to develop system here and now. It furthermore identifies weaknesses of the current technology and opportunities for further basic research.

The mobile devices are connected over a wireless LAN. The Ekahau positioning engine calculates the location from W-LAN signal patterns with a precision of 3-5 meters. The system consists of a server and PDAs (IPAQs) as mobile clients. The system uses a replicated architecture: Every three seconds the client makes a full replication of his own state with the game state on the server. A more detailed description of the system architecture can be found in $[2,3]$.

\subsection{Description of major interface versions}

This section briefly introduces the major system versions. A detailed discussion of the rationale of the changes is reserved for subsequent sections. The first prototype was developed in 2003 as a technical feasibility study and tested in fall 2003. The user interface consists of four core areas (Figure 1):

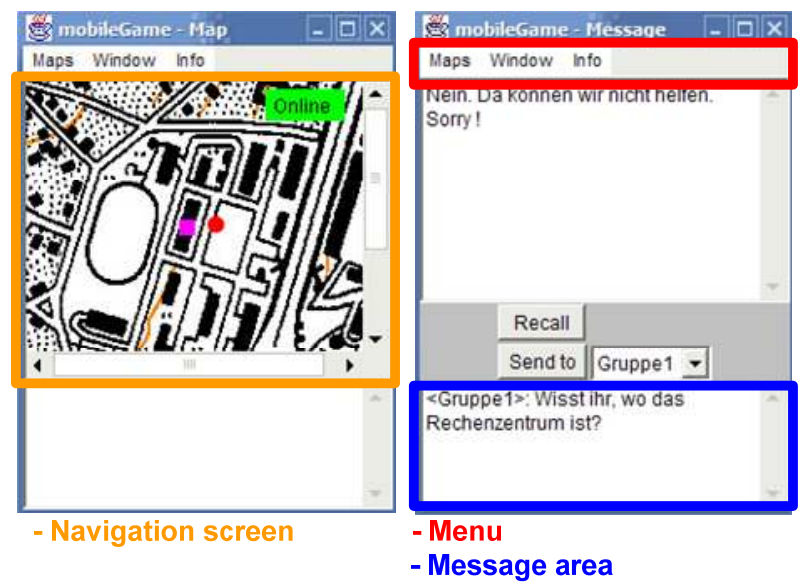

Figure 1: First Interface ${ }^{3}$

- Navigation area (Figure 1, left): This area contains the map, the position of the player, the position of the two direct competitors (hunter and prey), and the position of the current task. The position is indicated with a coloured round point (participant) or square point (task). There is no zoom and scrolling is manual with a PDA pen.

- Task area (no picture): This area contains information about the current task and space to solve it. The first version supported only open questions which where answered by entering text.

- Message area (Figure 1, right): The message area is similar to an instant messenger. Players can type in a message over the PDA's virtual keyboard and send it to another group or the game leader. A recall button allows to recall a sent message and to resend it to another group.

${ }^{3}$ Design by Christoph Göth 
The message area also contains system generated status messages.

- Capture-area (no picture): This area provides the players with information whom they hunt and who hunts them. If one player wants to capture another player, she has to go to his position and push the capture button.

Pop Up menus are used for navigation between areas. A subsequent intermediate version included the walking direction information Ekahau provides. However it was dropped without user tests because of its low reliability and jumping movements.

Building on the first user trials a new version was developed and tested in spring 2004 (Figure 2).

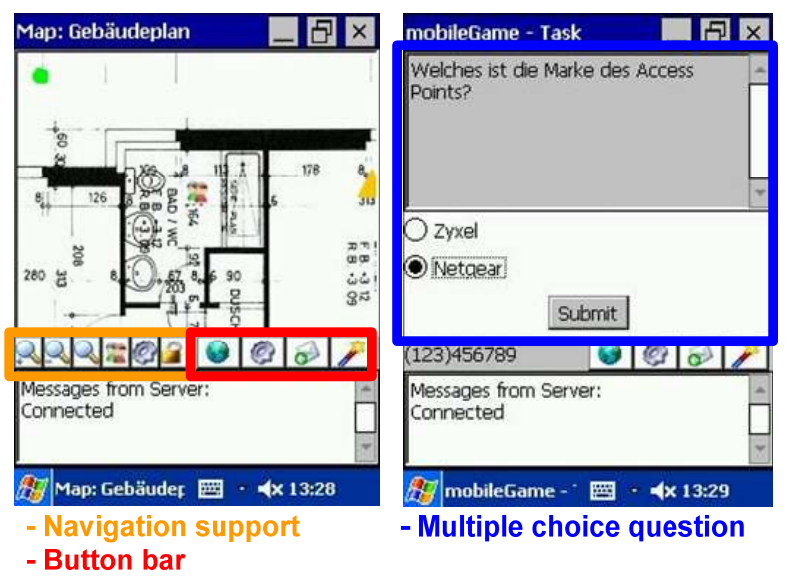

Figure 2: Second Interface ${ }^{4}$

This version was optimised for pen use: Pop-up menus were not accepted well and thus were replaced by a button bar allowing to switch between the areas with one click. Zoom and auto scrolling function (the map moves with the players position) were added to improve navigation. Positions of participants were indicated with icons instead of coloured dots to improve their visibility. The slide bar for manual scrolling was replaced by the option to directly move the map on the screen with the pen. Multiple choice questions and slider questions (for number input) were added for easier input of solutions.

In summer 2004 the third system version was developed. The major driver for the fast change was an improved Java version. The experiences in the prior user trials lead to a further improvement of the interface design (Figure 3). The Button bar was moved to the system bar on the bottom of the screen (this was not possible in prior Java versions). The message area of the prior version was split to a status bar (for status messages, visible in all areas) and a communication area on a separate screen.

${ }^{4}$ Design by Christoph Göth, Dr. Malgorzata Bugajska and Urs-Peter Häss
This facilitated enlarging the navigation area to nearly the whole PDA screen. An aura around a player's own location indicates the precision of the location information. The zooming and manual scrolling functions were moved to the PDA's scroll button. The stepless zoom was replaced by two zooming views (overview and detailed view). The rationale for those design decisions will be discussed in sections 4 and 5.

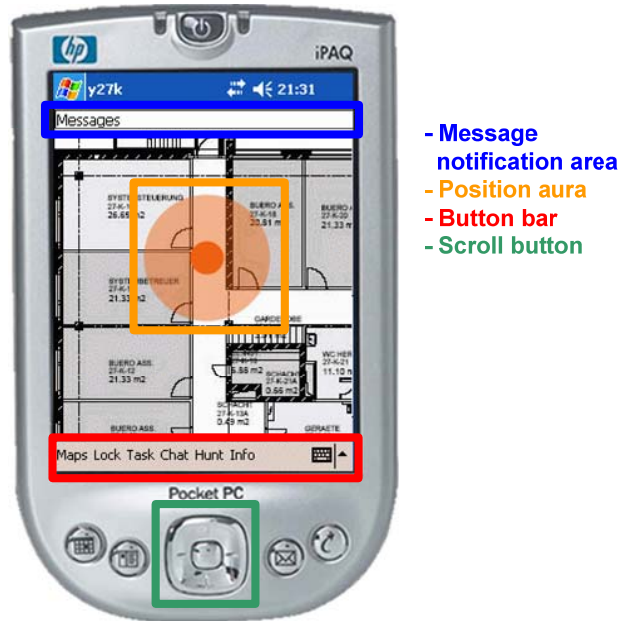

Figure 3: Third Interface ${ }^{5}$

\section{Research Design and Data Collection}

The overall design of MobileGame research follows the pilot study research approach [5]. Its basic idea is to develop socio-technical innovations in close collaboration with the field site and to iterate between development and evaluation activities. Sharples [6] adapted these ideas to the educational area and refined them. As mobile game based learning is an innovation, a scenario was as the starting point (for scenario based design see [7]). This scenario has been described in section 2.1. Following Taylor's [8] recommendation the first test focused on the basic usability of the technology, and the following steps on its motivational and learning effects (those effects are reported in [1]). After each step, the software was adapted to the newly surfacing requirements. The first test in October 2003 focused on the usability. Seven volunteering students were asked to use the system to find a hidden PDA. Then they completed a short questionnaire concerning their experience. There was a section with closed questions in which the participants evaluated technical aspects of the game and a section with open questions where the participants provided feedback regarding the game.

In May 2004 the second prototype was tested at the Koblenz Campus of the University of Koblenz-Landau in

${ }^{5}$ Design by Christoph Göth, Patrick Knab and Dirk Frohberg 
Germany. A total of 22 students volunteered to participate in one of two games (one with 13 participants and the other with 9). Eighteen participants played the game on their own with a device of their own. Four participants played the game with a partner and shared one device. The participants were videotaped while playing the game. The students were given 10 tasks, each consisting of a location they had to find, and a location related question to answer. Details on the design of this experiment and the data collection can be found in [1]. The questionnaire section relevant for this paper collected data on the usability of individual functions. It contained three parts: The questions of Brooke's general usability test [9], a set of specific usability questions and a part with open feedback to each of the systems major screens.

At the beginning of the winter term 2004, all 149 students of an introductory course to computer science were asked to participate in a test of the third prototype (this is a much higher number of users than by comparable studies like [10-12]). The game introduced them to the Irchel campus of the University of Zurich. There were 12 possible dates to participate in the game. Before each game run started, the students were given a pre-questionnaire with questions to their personal data and six knowledge questions about the campus. Afterwards, there was a short training session and then the game started. Each game lasted approximately 45 to 50 minutes $^{6}$. In this time, the students had the task to navigate to significant campus locations and to answer as many of 12 location-specific questions as possible. While doing so, they could catch other teams (and gain points) and communicate with other teams. The questions were distributed in a random order. At the end of each game, each player was given a first post-questionnaire. The first post-questionnaire contained questions about their general impression and the six knowledge questions from the pre-questionnaire. The participants were also asked to fill in an extended second post-questionnaire at home as soon as possible and return it one week later. The extended post-questionnaire contained more detailed questions on the participants' experience and their evaluation of the software features.

There was a maximum of 18 persons and a minimum of 5 persons participating in each game run. In each game, there was a maximum of 9 teams and a minimum of 3 teams. There were single players with one PDA and teams of two, three, four and five sharing one device. An analysis shows that the performance of the user groups differs significantly with team size [13]: Teams of two performed best and a team size of larger than three cannot be recommended. The analysis of this paper is based only on the 70

${ }^{6}$ The short duration is mostly caused by limited battery power of the IPAQs users in teams of two and 9 individual users. Due to technical problems in the server, log data is missing for some teams. We also excluded one team from all evaluations (team level and individual level) who had started with two persons and only one person came back. All other participants handed in a short post-questionnaire filled in directly after the experiment. The majority of the participants also handed in the second post-questionnaire - some of them after reminders.

A final small fourth test was undertaken in March 2005. The test was based on an unchanged prototype and explicitly focused on identifying requirements for an improved navigation interface. The groups with a total of seven student participants played the game for 45 minutes and met afterwards for a 60 minute electronic meeting using GroupSystems to gather and prioritise requirements for an improved navigation interface.

\section{Designing Maps and Map interaction}

The navigation experience contributes largely to the fun players have in the MobileGame. In the second trial, participants rated the dynamic position tracking on a map as most important value added for an orientation game [1]. The provision and visualisation of dynamic location information raises major design issues: How to design the map, how to interact with the map, how to deal with imprecise location information and how to deal with time delays.

Map Design: The creation of high quality digital maps for large sections of a university campus can become prohibitively expensive. Economy forces the game designers to start with maps available. The Ekahau positioning engine requires scaled maps. In Zurich architectural maps and emergency evacuation maps were available. The first two prototypes were based on architectural maps. In the second trial 10 of 28 suggestions for improving the navigation requested an improved map. Architectural maps provide too much information (e.g. the size of a room) and too little information (e.g. the function of a room) at the same time. Some information is furthermore heavily coded (e.g. room codes like Y24-K26). The small screen size amplifies the users' problems. The third trial used emergency evacuation maps for the core areas of the game. As emergency evacuation maps are optimised for quick user orientation, they provide the right set of information. The users responded positively to them and the remaining complaints focused on the areas covered by architectural maps and on the interaction with the map.

Design recommendation 1: Base the navigation interface on maps designed for walking.

Interacting with maps: Already the first test user requested zooming and auto scroll. Zooming allows the user to choose the level of detail on the screen and auto scroll 
assures that the direct surroundings of a player are always visible on screen. There was contradictory feedback on the appropriate manual scrolling and zooming. In the second trial the participants used a pen to scroll and zoom. They rated this interaction as too difficult $\left(3,33^{7}\right)$. In the third trial the participants used the PDA-buttons to scroll manually and to choose between two pre-set zoom levels (overview and detailed view). Again they were not happy $\left(3,78^{8}\right)$. The zoom levels in particular were regarded as too coarse. This contradiction lead us to the conclusion that there are two different phases for navigation interaction: While participants are on the move, fast use is paramount and while they are standing (e.g. during or after solving a task) they require a more fine-grained map interaction. Thus it is appropriate to offer both functionalities.

Design recommendation 2: Precise zooming is adequate for standing use only; button based coarse zooming is adequate for walking.

Improvements: Both in the open user sections and in the fourth trial the users proposed a large number of ideas for improving map navigation:

- Maps should have different layers of information. The users can then choose what information they want to see.

- An electronic compass should always show North or the map should be aligned to the viewing axis of the user.

- Electronic markers should indicate places already visited and thus link map locations with the participant's memory. These markers could be represented as icons or coloured sections.

- The users should be able to plan a path and to store this plan on the map.

- A map may give some guidance information over (computer generated) voice, e.g. "turn right". The problems of this approach have been discussed above.

Design recommendation 3: Good navigation support does not only cover the current situation, but also captures the past and supports planning.

Dealing with imprecise location information: Indoor navigation requires a higher precision than outdoor navigation. The Ekahau system provides positioning information with a reliable accuracy of $3-5 \mathrm{~m}$. The effect of the limited accuracy is amplified by information delays. The tested prototypes update the location information every three seconds and - due to time delays of the Ekahau server - in the worst case the location information is up to 6 seconds old. The participants rated the update time with

\footnotetext{
${ }^{7}$ Points on a scale from $1=$ very bad and $5=$ very good, $\mathrm{N}=22$

${ }^{8}$ Points on a scale from $1=$ very bad and $5=$ very good, $N=67$
}

$2,88^{9}$, which means that it is just about acceptable. About half of the time delays can be removed by improving the MobileGame architecture, but the other half have to be accepted due to limitations of the Ekahau engine and PDAs. Thus there is an important design issue how to present the imprecise location information to the user. For physical reasons, the location error of the Ekahau engine is not linear; i.e. the Ekahau engine may interpret a small change of physical location as a large step. A direct implementation of location information produces a small location arrow that jumps over the map. Guessing the walking direction is also not precise, and the arrow changes direction in an unpredictable manner. In a sense it is interesting to observe that such a navigation system was still regarded as usable by the users of the second trial (they rated the positioning part of the game with $4,23^{10}$ ). If one wants to look at design solutions one has to distinguish three basis activities:

1. Navigation to another location: Here it is most important to indicate the precision of the location information to the users. The third prototype did so by replacing the "dancing arrow" with a circle with an aura covering the area, in which the person very likely really is. The test users responded favourably to this feature. The participants of the third trial rated the navigation functionality with $4,58^{11}$; an improvement of 0,35 compared to the second trial. Its usability was rated $3,72^{12}$. A further improvement could be a dynamic aura indicating the real imprecision instead of the maximum imprecision of a static aura.

2. Solving a task: Here the participants reported most: A task requires the participant to be in a certain area. Small physical movements may move participants outside this area (due to the non-linear behaviour of Ekahau) taking away the task from the participants. An appropriate design answer is an automatic or manual locking of location information while a participant is solving a task. If a task requires local movements beyond the precision of the W-LAN positioning system, changing to a Personal Area Location Information system with active digital objects (e.g. based on RFID or Bluetooth technology) is advisable.

3. Catching and being caught: Hunters catch their prey by coming into their close proximity. Players reported that the low precision makes it difficult to calculate the danger a player is in. A possible design solution would be to visualise a danger zone, but this has to be carefully designed in order to avoid spoiling the fun.

\footnotetext{
${ }^{9}$ Points on a scale from $1=\mathrm{I}$ was very unsatisfied and $5=\mathrm{I}$ was very satisfied, $\mathrm{N}=69$

${ }^{10}$ Points on a scale from $1=$ very bad and $5=$ very good, $\mathrm{N}=22$

${ }^{11}$ Points on a scale from $1=$ very bad and $5=$ very good, $\mathrm{N}=69$

${ }^{12}$ Points on a scale from $1=$ very bad and $5=$ very good, $\mathrm{N}=69$
} 
Design recommendation 4: Visualise imprecision of location information appropriately.

Car navigation for walkers: All three MobileGame versions are based on the notion that the users find their way using an electronic map (as this is a learning objective). Note that this concept prohibits the direct transfer of car navigation to game navigation. It may also conflict with the fun of the game. In the second and third trial the users rated the map navigation one of the most enjoyable aspects of the game (second trial 4,23 and third trial $4,58^{13}$ ). Users are drawn into a mixed reality experience. This experience may not be the same any more if the navigation activities are moved from the participants to the machine.

\section{Channel Usage}

The MobileGame offers four major channels: A navigation channel, a channel for task interaction, a channel for communication and a channel for providing social awareness (e.g. on the activities of others, their gamepoints etc.). In each channel, there is a continuous flow of independent information. It is a major design challenge to arrange the channels in an appropriate way. The user trials clearly indicate that the navigation channel is dominating, i.e. it is the default activated channel from which participants deviate for a certain purpose and then return. The next paragraphs will therefore discuss how to arrange the other channels in comparison to the navigation channel and how to design them.

Navigation vs. Task interaction: A typical game task consisted of three parts: To plan a way to a specific location on the map, to go there and then to execute the task there. Thus the participants typically needed at any given time either navigation information, or task specific information. Already in the initial design the PDA represented navigation and task interaction on different screens and the design focused on easy channel switching.

The design of the task interaction itself is difficult: Allowing for open questions can make the game much richer and more challenging. However, the users have to write down the answer with the pen and a small virtual keyboard on the PDA, which is difficult to use. Multiple choice questions restrict the game design and are said to be didactically poor, but users can answer with a simple click. Both the spring 2004 evaluation and the fall 2004 evaluation show that the users liked the multiple choice questions. Frequent complaints in the third trial indicate that the users rather disliked the open questions ${ }^{14}$. This

${ }^{13}$ Points on a scale from $1=$ very bad and $5=$ very good, second trial $\mathrm{N}=$ 22, third trial $\mathrm{N}=69$

${ }^{14}$ There were 26 comments to the open task screen. Seven of them complained about the question type and 15 complained about the text input supports our notion that users like to use the system as a "machine" rather than as a "tool" [14]. It is an open question whether a better communication channel (e.g. using voice) could also be used for task execution.

Navigation vs. communication: The MobileGame offers the participants the opportunity to chat with specific other groups, the plenary or the game leader. The game designers expected several advantages of such a feature:

a) Different groups could help one another solving tasks. From a point of view of competition it makes sense to form alliances that both groups benefit from. It was hoped to enhance learning this way.

b) Different groups could socialise over the communication tools (e.g. exchange jokes, challenge others) increasing the fun for all participants and finally improving team building between all participants.

c) The game could become more exciting for the participants and bystanders if the "noise level" increases. Such an effect has been reported by Benford [11].

However only a minority of the participants used the chat communication features although it was regarded as moderately useful (in the third trial it was $\left.3,48^{15}\right)^{16}$. Reasons may be just lack of time or incentive. But there are also four interface-related reasons. 1. Typing in questions is not user-friendly with IPAQs. A better mobile keyboard may help here. 2. Communication is typically a process accompanying navigation and task execution. Thus it should be preferable to see both the communication channel and the navigation/task execution at the same time. The first prototype was designed according to this rationale. This would lead to an insufficiently small navigation space. Later prototypes moved the communication to a separate screen. This did not influence usage, but it is clearly not the optimal solution. 3 . Writing chat messages does not create an interesting noise that raises excitement. 4. Under time pressure the benefits of communication do not outweigh its costs. In comparison to oral communication, chat has a lower degree of synchronicity [15]: Communication is slightly delayed and therefore feedback is slower. The higher persistence allows a higher degree of parallel communication. Under time pressure, fast feedback is more important than parallel, persistent channels. Furthermore, oral and written communication has different cost functions (in terms of time spent): Writing is slower than talking, but reading faster than hearing. Therefore, ceteris paribus, writing benefits more the consumer and talking the producer of information.

and four participants remarked explicitly that they liked multiple choice better, although we did not ask for a comparison.

${ }^{15}$ Points on a scale from $1=$ very bad and $5=$ very good, $\mathrm{N}=69$

${ }^{16}$ As a sidenote: Single users rated the chat functionality significantly better than pairs of two. 
Design recommendation 5: Chat-based communication is inadequate for many game situations. Carefully designed oral communication should be more adequate in many situations.

Thus, in its current form, the chat feature was rather a break than an accelerator. In both 2004 evaluations participants explicitly asked for voice communication. Voice communication could solve those four problems. It is easier to enter, it can be used in parallel with navigation, it is easier to create interesting "noise" and it is highly synchronous. Such a voice channel needs to be carefully designed as one can expect different usage for standing and moving participants: standing participants typically focus on task solution and will need specific information. A one-to-one phone conversation is the most appropriate way to gain that information. They may keep chat as a useful backup-channel. A moving participant has no use for chatting; they typically desires to share their excitement, crack a joke or get some help in navigation. Broadcast information can be very useful to raise the excitement level, as observed in the Can-You-See-Me-Now Game [11]. One-to-many oral communication (like with a walky-talky) should be an appropriate way to reach that means.

Competitive awareness: The rules of the MobileGame are designed to a competitive environment. Competition only works if it is transparent to the players.

1. They have to know how well they do in comparison to others (particularly: how many points they have),

2. they have to be able to react to competitive threats (being caught by others) and

3. they have to know important events.

In contrast to the discussed three channels, competitive awareness information does not need a specially designed screen. Rather, the information is distributed to several screens: Awareness on the location of hunter and prey are integrated in the navigation map. A separate hunting screen is only needed for more detailed information and the act of capture itself. In the first two versions the notification service was integrated in the communication channel. As notifications have to be visible all the time, this was not possible any more once the communication channel was removed from the navigation screen. A message line has successfully taken its place. The game status is also presented on a separate information screen. Standing players have different competitive awareness needs than moving players: While standing they need the complete set of competitive awareness information; while moving only the most urgent status messages and the location of other relevant actors is needed.

Design recommendation 6: Embed competitive awareness into the other channels.
Summary of difference for stationary and motionary use: There are different requirements for using the systems when stationary and when being on the move. As they have been completely discussed by now, Table 1 summarises the requirements discussed in prior sections.

Table 1: Requirements for stationary and moving use

\begin{tabular}{|l|l|l|}
\hline & Requirements Stationary & $\begin{array}{l}\text { Requirements on the } \\
\text { move }\end{array}$ \\
\hline Navigation & $\begin{array}{l}\text { precise navigation: pen } \\
\text { input, precise zooming; } \\
\text { indicators on precision of } \\
\text { location information, sta- } \\
\text { ble positioning }\end{array}$ & $\begin{array}{l}\text { quick navigation: but- } \\
\text { ing; indicators on pre- } \\
\text { cision of location in- } \\
\text { formation }\end{array}$ \\
\hline $\begin{array}{l}\text { Communi- } \\
\text { cation }\end{array}$ & $\begin{array}{l}\text { voice plus chat; one to one } \\
\text { communication }\end{array}$ & $\begin{array}{l}\text { voice communication } \\
\text { only, one to many }\end{array}$ \\
\hline $\begin{array}{l}\text { Task inter- } \\
\text { action }\end{array}$ & $\begin{array}{l}\text { information on task, no } \\
\text { complex written input: } \\
\text { multiple choice, phrases, } \\
\text { hand-over e.g. of photo or } \\
\text { voice file }\end{array}$ & $\begin{array}{l}\text { not possible currently, } \\
\text { specific asks are location }\end{array}$ \\
\hline $\begin{array}{l}\text { Competi- } \\
\text { tive } \\
\text { awareness }\end{array}$ & $\begin{array}{l}\text { location of other partici- } \\
\text { pants, further information } \\
\text { on other participants acti- } \\
\text { vities (current and past ), } \\
\text { status information }\end{array}$ & $\begin{array}{l}\text { location of other par- } \\
\text { ticipants only, urgent } \\
\text { status information }\end{array}$ \\
\hline
\end{tabular}

Design recommendation 7: Design explicitly both for standing and motionary use.

Multi-user, multi-channel management: Data from the third trial clearly shows that: Single users were using the system significantly less successfully than teams of two: They solved significantly fewer tasks than teams of two $\left(2,77\right.$ vs. 4,41 tasks $^{17}$, sig. $\left.=0,048<0,05\right)$ and they rated the general usability of the system (on Brooke's scales [9]) significantly lower $\left(63,50\right.$ vs. $75,85^{18}$, sig. $\left.0,056<0,1^{19}\right)$. Single users rated the navigation functionality significantly lower than the members of teams of two $\left(2,60\right.$ vs. $3,81^{20}$, sig. $\left.=0,047<0,05\right)$.

We interpreted this difference with a cognitive overload: MobileGame involves complex physical navigation, complex social interaction, and a complex task at the same time. Players may have to deal simultaneously with information coming from different channels. This cognitive load may hamper the performance of an individual, particularly if the information quality varies. One may argue

\footnotetext{
${ }^{17}$ Points on a scale from $1=$ very bad and $5=$ very good, individual $\mathrm{N}=9$, teams of two $\mathrm{N}=34$

${ }^{18}$ Points on a scale from $1=$ very bad and $5=$ very good, individual $\mathrm{N}=$ 6 , teams of two $\mathrm{N}=63$

${ }^{19}$ Significance is measured with a one-sided t-test. As there were only 9 individual participants, $90 \%$ reliability is regarded as acceptable

${ }^{20}$ Points on a scale from $1=$ very bad and $5=$ very good, individual $\mathrm{N}=$ 6 , teams of two $\mathrm{N}=63$
} 
that this is due to poor interface design. However both authors have extensive experience in running conventional (paper based) orientation games. Here we have observed the same difficulties. Thus the difficulties are primarily due to the nature of the task. Even in conventional games, groups typically delegate navigation tasks to two persons. Pairs of two distribute the load between two persons. They help one another with navigation, particularly matching the real world with the map world. As many people can better think when they talk, making issues explicit in conversation with partners helps to resolve problems and gains self-assurance.

Design recommendation 8: Design systems for user pairs.

The team-members may furthermore split channels, e.g. one person is in charge of navigation and hunting and the other is in charge of task fulfilment and communication. The higher success of two leads to a new set of open research questions: How should a system look that supports two players? Should there be two or more devices for them? If yes, how should they be linked? Or can channels be designed and arranged so simply that the advantages of playing alone prevail?

\section{Summary and Implications}

The iterative design and test approach turned out to be fruitful as it provided a deeper insight into major design issues of a mobile learning game. Some of those design lessons can be transferred to other sectors that combine navigation with activity and communication. These sectors range from tourism, first aid, facility management, cleaning services, large fairs to museum visitors. Lessons that can be transferred include:

1. The distinction between standing and moving users helps to identify situation based user requirements and supports the idea of fading in more detailed information if the situation requires it.

2. Supporting pairs of users may be a more appropriate approach to reduce complexity than developing too simplistic systems for single users.

3. As long as location information is imprecise, the discussed visualisation and locking approach may help improve the usability of mobile systems.

4. The more functions are combined into one device the more fruitful may be a thorough, user-oriented analysis of appropriate channel combinations.

We are currently working on a fourth version of MobileGame and are curious what we will learn once we run trials with it.

\section{REFERENCES}

[1] G. Schwabe and C. Göth, "Mobile Learning with a Mobile Game: Design and Motivational Effects.," Journal of Computer Assisted Learning, 2005.

[2] C. Göth, "Prototypische Implementierung einer mobilen Spielumgebung für den PDA," in Institute for IS Research. Koblenz: University of Koblenz-Landau, 2003.

[3] C. Göth, U.-P. Häss, and G. Schwabe, "Requirements for mobile learning games shown on a mobile game prototype," in Proceedings of the MLearn2004. Rome, Italy, 2004.

[4] Mobilearn, "http://www.mobilearn.org."

[5] G. Schwabe and H. Krcmar, "Piloting a Sociotechnical Innovation," in Proceedings of the 8th European Conference on Information Systems ECIS 2000. Wien:

Wirtschaftsuniversität Wien, 2000, pp. 132-139.

[6] M. Sharples, N. Jeffery, J. B. H. du Boulay, D. Teather, B. Teather, and d. B. G. H, "Socio-cognitive engineering: a methodology for the design of human-centred technology," European Journal of Operational Research, vol. 136, pp. 310-323, 2002.

[7] J. Caroll, Scenario-Based Design: Envisioning Work and Technology in System Development. Indianapolis: Wiley \& Sons, 1995.

[8] J. Taylor, "A Task-centred Approach to Evaluating a Mobile Learning Environment for Pedagogical Soundness.," in Presentation at the Mlearn. Birmingham, 2003.

[9] J. Brooke, "SUS - A quick and dirty usability scale," in Usability Evaluation in Industry, P. W. Jordan, B. Thomas, B. A. Weerdemeester, and I. L. Mc Clelland, Eds. London: Taylor \& Francis, 1996, pp. 189-194.

[10] H. Balakrishnan, R. Baliga, D. Curtis, M. Goraczko, A. Miu, N. B. Priyantha, A. Smith, K. Steele, S. Teller, and $\mathrm{K}$. Wang, " Lessons from Developing and Deploying the Cricket Indoor Location System," Preprint, 2003.

[11] S. Benford, R. Anastasi, M. Flintham, C. Greenhalgh, N. Tandavanitj, M. Adams, and J. Row-Farr, "Coping with uncertainty in a location-based game," Pervasive Computing, IEEE, vol. 2, pp. 34, 2003.

[12] A. D. Cheok, W. Fong Siew, Y. Xubo, W. Wang, H. Lee Men, M. Billinghurst, and H. Kato, "Game-City: a ubiquitous large area multi-interface mixed reality game space for wearable computers," in Proceedings to the International Symposium on Werable Computers, 2002, pp. 156.

[13] G. Schwabe, C. Göth, and D. Frohberg, "Does Team Size Matter in Mobile Learning?" in Proceedings to the International Conference on Mobile Business. Sydney,

[14] BOBsudde and H. Züllighoven, "Software Tools in a Programming Workshop," in Software Development and Reality Construction, C. Floyd, H. Züllighoven, R. Budde, and R. Keil-Slawik, Eds. Berlin, Heidelberg, New York. Tokio: Springer-Verlag, 1992.

[15] A. Dennis and J. Valacich, "Rethinking Media Richness: Towards a Theory of Media Synchronicity," in Proceedings of the 32nd Annual Hawaii International Conference on System Sciences, 1999. 